\title{
Implementation of a novel eccentric dog leg reamer in oil well drilling
}

\author{
Amir Shokry ${ }^{1}$ (D) Ahmed Elgibaly ${ }^{2} \cdot$ Adel Salem $^{2}$ \\ Received: 16 September 2020 / Accepted: 18 January 2021 / Published online: 4 March 2021 \\ (c) The Author(s) 2021
}

\begin{abstract}
A high-quality wellbore is generally considered to have a gauge hole, a smooth wellbore and a wellbore with minimum tortuosity. Therefore, dog leg reamers (DLRs) have been recently proposed and implemented to specifically provide a high-quality wellbore which in turn improves the overall drilling performance. These improvements include smoothening the wellbore from high in place dogleg, lowering drag values, improving the tripping performance, increasing the success ratio of wireline logging tools to reach total depths and enhancing the casing and cement jobs. DLRs are an eccentric string reamers which have been recently designed and optimized using modelling software, and a mathematical model has been deduced to specify the weight and torque distribution between the bit and DLR based on mechanical specific energies at bit and DLR with a model accuracy closely matches the field data. A total of twenty five runs all over the world have been performed to date in hole sizes ranging from 121/4" to $6 "$. Directional work has also been performed showing good directional control with this system, and no vibration issues have been reported. The development of this down hole tool has added new capability to the industry in the optimization of hole enlargement applications. The benefit is realized in a reduced number of days required to drill the well and in reduced total well cost. In addition, getting casing into the hole sooner reduces the accompanying risks and can contribute to reducing operational nonproductive time. Several case studies from three fields $X, Y$ and $Z$ from Egypt, Oman and Saudi Arabia, respectively, will be discussed to demonstrate the positive economic impact of deploying DLR in not only the deviated holes but also in vertical wells.
\end{abstract}

$\begin{array}{ll}\text { Abbreviations } \\ \text { TD } & \text { Total depth } \\ \text { RSS } & \text { Rotary steerable system } \\ \text { DLR } & \text { Dog leg reamer } \\ \text { BHA } & \text { Bottom hole assembly } \\ \text { NPT } & \text { Non-productive time } \\ \text { BHE } & \text { Bore hole enlargement } \\ \text { PDC } & \text { Polycrystalline diamond compact } \\ \text { ROP } & \text { Rate of penetration } \\ \text { WOB } & \text { Weight on bit } \\ \text { RPM } & \text { Revolution per minute } \\ \text { Fc } & \text { The circumferential force applied to DLR }\end{array}$

Amir Shokry

petrol_eng_amir@yahoo.com

$\triangle$ Adel Salem

adel.salem@suezuni.edu.eg

Ahmed Elgibaly

elgibaly.A@yahoo.com

1 Badr El-Din Petroleum Company, Faculty of Petroleum and Mining Engineering, Suez University, Suez, Egypt

2 Faculty of Petroleum and Mining Engineering, Suez University, Suez, Egypt
Fp The radial force applied to DLR

Fr The resultant force applied to DLR

POOH Pull out of hole

MSE Mechanical specific energy

ID Internal diameter

TOB Torque on bit

TOR Torque on reamer

WOR Weight on reamer

HWDP Heavy weight drill pipe

\section{Introduction}

Simultaneous hole enlargement while drilling, also known as reaming while drilling, is a practice of initiating a hole with a smaller-diameter pilot bit and simultaneously enlarging it with a borehole enlargement (BHE) device placed behind the pilot bit. The reasons for BHE vary from one application to another (Chowdhury et al. 2019).

BHE tool is defined as a tool that can expand a borehole beyond the diameter of its pass through diameter. This allows to create a larger borehole than the previous in place 
casing string. BHE tools can be classified into two primary categories: eccentric and concentric.

BHE and specifically under reaming has become a wellestablished practice in the drilling industry and can be used to successfully overcome numerous wellbore stability issues and provide flexibility to casing/completion programs (Huggett et al. 2019).

An eccentric BHE tool is defined as a tool with its maximum diameter smaller than twice its maximum radius, as measured from the centre of the drill string. This allows the tool to pass through a restriction equal to its diameter and create a hole with a diameter equal to twice its maximum radius, when rotated about the centre of the drill string. The tool does this by describing a radius about the geometric centre of the drill string that is larger than the radius of its pass through size. Its eccentricity works by being stabilized in a smaller pilot hole.

A concentric BHE tool is defined as an expandable tool that has a maximum diameter equal to twice its maximum radius, when measured from the centre of the drill string. The concentric has cutting structure that can be extended beyond the nominal diameter of the tool to enlarge the wellbore. These types of tools use differential pressure and/or weight on bit to provide the required energy to expand the cutting structure (Chowdhury et al. 2019).

The idea of using eccentric BHE concept to eliminate any tight spots, smooth micro-doglegs, agitate the cutting beds in directional application and overcome the different issues from swelling shales or soft Limestone has not been implemented before.

In this paper, we aim to explore the success story of DLR which depends mainly on the eccentric concept considering:

- Tool features, benefits and its design philosophy.

- Key factors for DLR performance optimization.

- Case studies for proper implementation of DLR tool starting from vertical wells to highly deviated wells.

- Mathematical model for torque and weight distribution between the drilling bit and DLR.

\section{Field application}

The DLR is an eccentric string reamer designed for maximum toughness. A key feature of this tool is the slightly eccentric profile which allows for an undergauge passthrough. When drilling a slightly larger wellbore, the tool assists in eliminating the tight spots, micro-doglegs and agitates the cutting beds in directional application, or issues from swelling shales/Soft Limestone or creeping salts.

\section{DLR benefits:}

- A slightly enlarge hole size reduces problems with setting casing or production liner.
- Can be used when drilling forward, backreaming while making connections or tripping out of the hole.

- Reduced trip time/reaming time.

- Eliminates the dedicated stiff reaming run/wiper trip.

- No effect on directional operations.

- Added benefit of stirring cutting beds.

- Allows fishing of most retrievable components.

\section{DLR applications:}

- Shale laterals.

- Extended reach wells.

- Cutting beds.

- Creeping salts.

- Swelling shales.

- Tight spots.

\section{DLR features:}

Forward spiral blades (Fig. 1).

- Adding a measure of protection, stability, durability and enabling drill out.

- Spiral blades are designed for both standard drilling and backreaming.

- Simple and robust design.

Eccentric design (Fig. 2).

- Allows tool to pass through an ID casing.

- Allows sliding behind bit.

- Drills larger diameter holes.

- Steel blades contact casing to prevent damage.

- Minimizes micro-doglegs more efficiently than a concentric design.

Torque control design (Fig. 3).

Using torque control components has many benefits:

- Prevent over-engagement of cutters into the formation.

- Maintain a gauged hole.

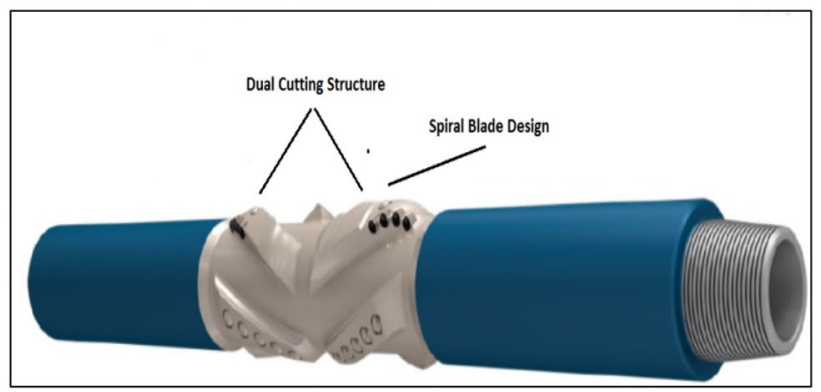

Fig. 1 DLR forward spiral blade feature 


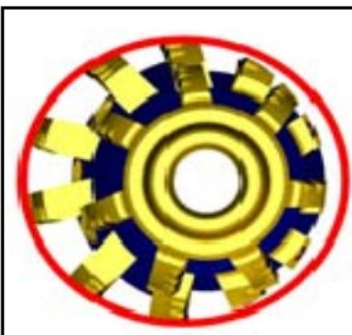

Eccentric Tool Pass Through

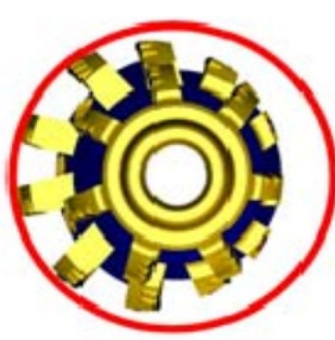

Eccentric Tool Drill Size

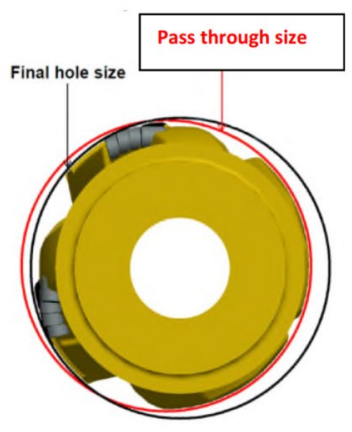

Fig. 2 DLR eccentric design feature (McCarthy et al. 2011)

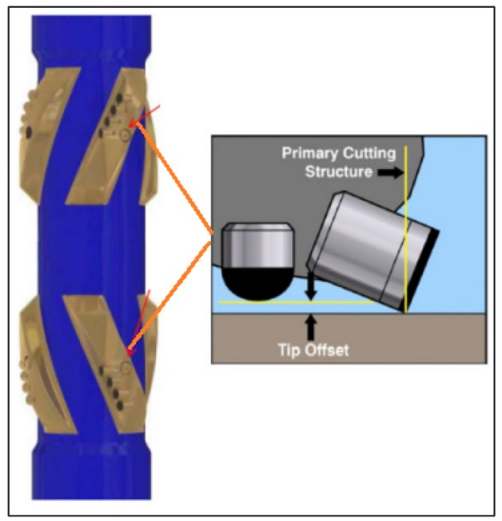

Fig. 3 DLR torque control feature

- Maintain homogenous ROP.

- Control lateral engagement.

- Improve lateral stability.

- Provide additional cutter protection.

DLR is normally used to drill out the shoe track without any challenges unlike the available known eccentric BHE that have limitation for doing that due to their very large expansion ratio over the pilot bit size, which is usually $50 \%$ but as shown in the below table (Table 1), DLR total enlargement is negligible compared to the pilot bit size, and this enables us to drill the section in one run from shoe to shoe.

Understanding the applied forces on DLR is very important to select the suitable DLR for the encountered drilling
Table 1 DLR available sizes

\begin{tabular}{lll}
\hline Hole Size & $\begin{array}{l}\text { Tool size } \\
(\text { Drift } \times \text { Drill Diameter })\end{array}$ & Total enlargement \\
\hline $171 / 2^{\prime \prime}$ & $173 / 8^{\prime \prime} \times 177^{\prime \prime}$ & $4 / 16^{\prime \prime}$ \\
16 & $157 / 8^{\prime \prime} \times 16^{1 / 4^{\prime \prime}}$ & $4 / 16^{\prime \prime}$ \\
$121^{\prime \prime}$ & $121 / 8^{\prime \prime} \times 127 / 16^{\prime \prime}$ & $3 / 16^{\prime \prime}$ \\
$81 / 2^{\prime \prime}$ & $83 / 8^{\prime \prime} \times 85 / 8^{\prime \prime}$ & $2 / 16^{\prime \prime}$ \\
$61 / 8^{\prime \prime}$ & $6 \times 63 / 16^{\prime \prime}$ & $1 / 16^{\prime \prime}$ \\
$6^{\prime \prime}$ & $57 / 8^{\prime \prime} \times 61 / 16^{\prime \prime}$ & $1 / 16^{\prime \prime}$ \\
$57 / 8^{\prime \prime}$ & $53 / 4^{\prime \prime} \times 515 / 16^{\prime \prime}$ & $1 / 16^{\prime \prime}$ \\
\hline
\end{tabular}

environment. System durability is an important factor in the success story of any DLR run. Both the cutting element and bottom hole assembly (BHA) need to be modelled and optimized for maximum durability by the means which not negatively affecting drilling efficiency (Barton and Wedeen 2010).

The placement position of the DLR in the BHA is a main concern as it can be placed directly above the directional tool in the compression part of the BHA or above the neutral zone in the tension section.

A comprehensive analysis can identify the optimum place for DLR based on DLR specifications, BHA configuration, well survey, mud parameters, expected drilling operating conditions, casing \& hole friction factors, previous casing depth and other factors to select the optimum placement for achieving the maximum output without any type of failure.

Based on software outputs and actual case studies, it is highly recommended to place the DLR away from the neutral zone in the tension zone and below are a set of charts comparing the contact forces and bending stresses in the drill string at the end depth for $X$ well for the tool placement analysis. The figures below show the results both without and with the DLR tool in the drill string based on the tool placement recommended location. The locations of the DLR tool are highlighted in green on the charts (Figs. 4 and 5).

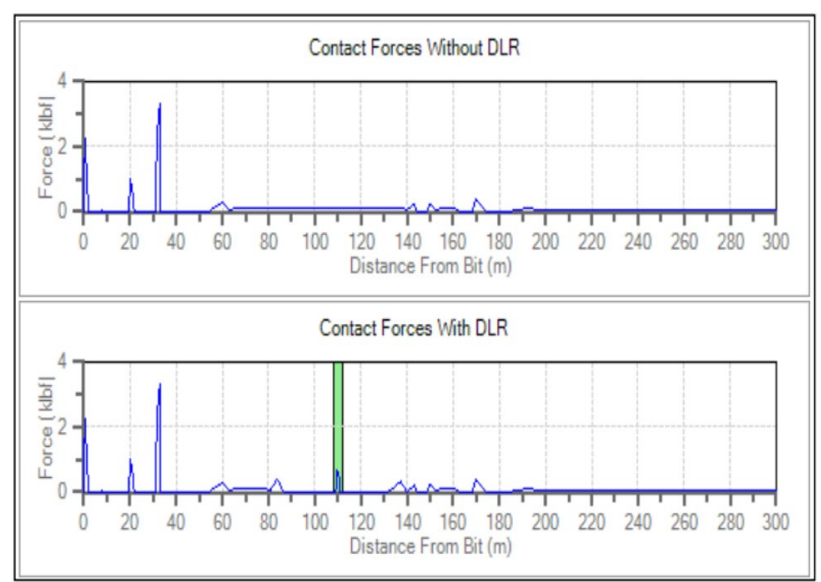

Fig. 4 Change of contact forces due to DLR 


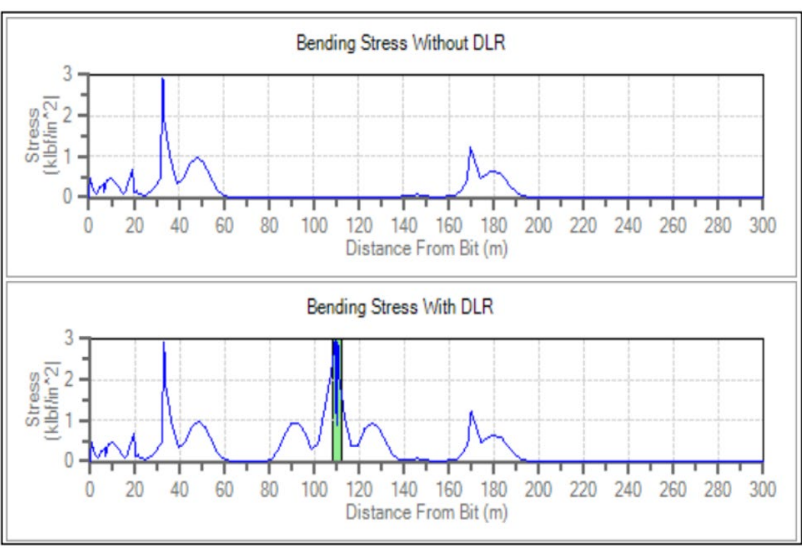

Fig. 5 Change of bending stress due to DLR

It is common to see an increase in the contact force magnitude at the location of the DLR tool, and this effect can be beneficial to the operation of the tool as these forces assist in engagement of the tool with the borehole.

Utilizing motor BHA to achieve the required directional work generates a borehole with an inconstant hole diameter with excessive doglegs and high tortuosity due to shifting between the sliding and rotary mode. This phenomena is significantly reduced when adding DLR to the BHA that produce a smooth hole.

Due to the eccentric motion of the DLR which is mainly dependent on the string RPM, it is recommended when deploying DLR with motor BHA to smooth the drilled interval, while the string is static in sliding mode by back reaming.

Smoothening the hole with RSS BHA or rotary BHA is a maturing drilling concept with notable successes reported due to the continuous rotation of the string. DLR can be utilized in all well trajectories irrespective of the amount of steering required, and it works successfully with both Pointthe-bit and Push-the-bit assemblies.

DLRs have been successfully deployed in some fields worldwide showing a significant performance. They proved their efficiency to smooth the well bore and minimize the wash and ream time during $\mathrm{POOH}$ as shown below in different case studies.

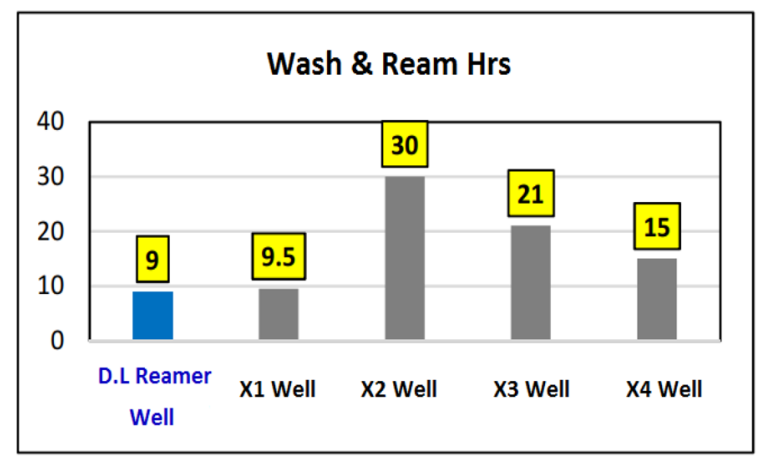

Fig. 6 W \& R Hrs of DLR versus field $X$ offset wells

DLR Proves Efficiency in its First Trial in Egypt Western Desert "Field X" in the first quarter of 2019 reducing Wash \& Ream NPT Saving \$12,000 compared to Direct Offset.

\section{Challenge:}

Drilling a vertical well achieving good borehole condition and minimize back reaming and eliminate wiper trips to reduce NPT.

\section{Solution:}

Using NOV $83 / 8^{\prime \prime} \times 85 / 8^{\prime \prime}$ DLR as an optimum solution to improve borehole quality and reduce tight spots that are often.

encountered which requires wiper trips in the middle of the section and after reaching TD resulting in back reaming and NPT. The DLR was utilized with a RSS BHA assembly placed $130 \mathrm{ft}$ above the bit drilling through Applonia, Khoman, A/R series, Bahariya \& Kharita formations (Table 2).

Outcome

- Saving $\$ 12,000$ compared to direct offset well (X3 Well) (Fig. 6).

- Saving \$21,000 compared to recent well (X2 Well) (Fig. 6).

- Saving $\$ 9,800$ compared to field average (Fig. 7).

- Pulled out of hole with minimal drag \& overpull observed
Table 2 Field $X$ recent wells against DIR Well

\begin{tabular}{lllllll}
\hline Well & $\begin{array}{l}\text { Depth In } \\
(\mathrm{ft})\end{array}$ & $\begin{array}{l}\text { Interval } \\
(\mathrm{ft})\end{array}$ & $\begin{array}{l}\text { ROP } \\
(\mathrm{ft} / \mathrm{h})\end{array}$ & $\begin{array}{l}\text { W\&R } \\
(\mathrm{h})\end{array}$ & BHA & $\begin{array}{l}\text { TQ } \\
(\mathrm{Kft} . l \mathrm{~b})\end{array}$ \\
\hline DLR & 3560 & 3125 & 104.5 & 9 & $\begin{array}{l}\text { RSS + 1 } \\
\text { Stab + DLR @ }\end{array}$ & $4-9$ \\
Well & & & & $\begin{array}{l}130 \mathrm{ft} \\
\text { RSS + 1 Stab }\end{array}$ & $2-6$ \\
X1 & 3512 & 2888 & 82.9 & 9.5 & RSS + 1 Stab & $2-6$ \\
X2 & 3615 & 2885 & 79.2 & 30 & Rotary + 2 Stab & $2-7$ \\
X3 & 3575 & 3230 & 126.7 & 21 & RSS + 2 Stab & $2-6$ \\
X4 & 3600 & 2900 & 84.3 & 15 & &
\end{tabular}




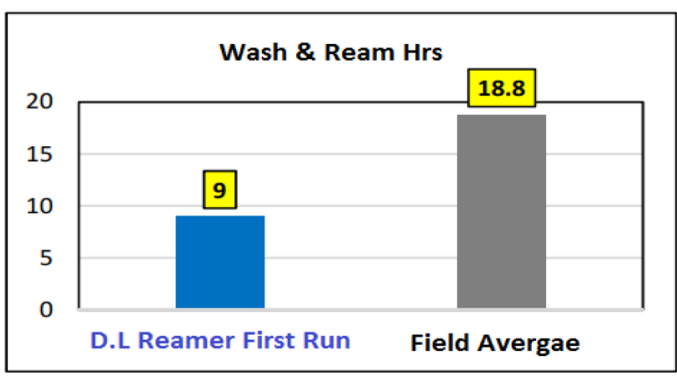

Fig. 7 W \& R Hrs of DLR versus Aver. $X$ Field

Another success using DLR in Oman "Field $Y$ " especially when placed at its right position at the BHA based on software analysis in the fourth quarter in 2019 reducing Wash \& Ream NPT Saving $\$ \mathbf{5 5 , 0 0 0}$ compared to direct offset.

Challenge

- Drill a vertical well achieving smooth borehole.

- Minimize the drag observed in offset wells.

- Enhance the life of MWD/LWD tools, protecting them from severe vibration levels while back ream (Table 2).

\section{Solution}

Using software analysis to select the best place for NOV $83 / 8^{\prime \prime} \times 85 / 8^{\prime \prime}$ DLR to achieve the maximum contact forces and this was as an optimum solution to improve borehole quality and reduce tight spots that are often encountered which requires wiper trips in the middle of the section and after reaching TD resulting in back reaming and NPT. The DLR was utilized with a Rotary BHA assembly placed $550 \mathrm{ft}$ above the bit.

\section{Outcome}

- Improve DLR performance when placed at the optimum place in the BHA showing a significant improvement against DLR Well \#3.

- Saving \$55,000 compared to direct offset Y1 Well (Fig. 8).

- Saving $\$ 26,000$ compared to field average (Fig. 8).

- POOH with minimal drag \& overpull observed (Fig. 9).

Another achievement using DLR in Saudi Arabia "Field Z" in two section wells in the first quarter of 2020 reducing Wash \& Ream NPT.

Challenge

- Eliminating the dedicated stiff reaming run prior to running the production tapered casing string.

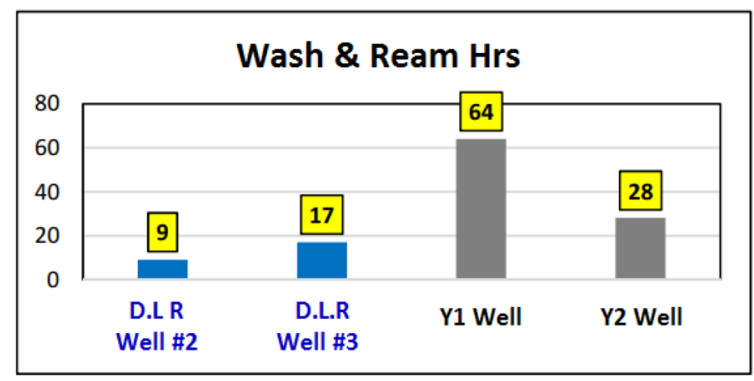

Fig. 8 W \& R Hrs of DLR versus field $Y$ offset wells

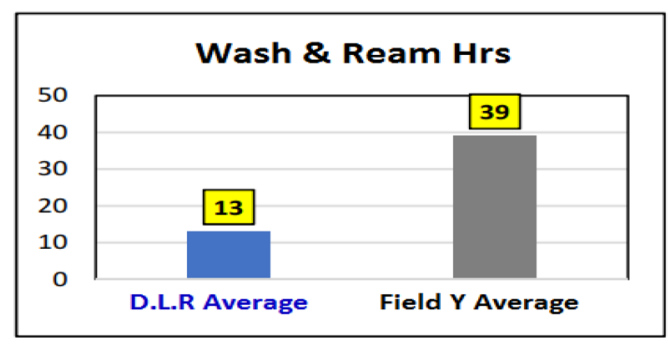

Fig. $9 \mathrm{~W} \& \mathrm{R}$ Hrs of DLR versus Aver. $Y$ field

- Minimizing reaming and back reaming hours and minimize open hole exposure time.

- Preventing stuck pipe events due to possible accumulation of cuttings bed and the existence of swelling shales in this long open hole section.

- Providing a smoother hole at the end to enable wire line logs to reach the bottom successfully.

\section{Solution}

DLR was proposed in the build/lateral section to enlarge/ smooth the borehole utilizing the bi-directional cutting structure design that allows the DLR to smoothen the hole while drilling ahead and while back-reaming. The eccentric design of the DLR creates a slightly enlarged hole and clears any tight spots which could have been created due to hole instability issues or well tortuosity (Table 3).

- First Run place the DLR on the motor BHA to drill the build section, the DLR tool was placed among the 5" HWDP's 203ft away from the bit.

- Second Run place the DLR on the SLB's Power Drive Orbit RSS BHA to drill the lateral section, the tool was placed among the 5" HWDP's 204ft away from the bit.

\section{Outcome:}

- DLR tool achieved its objectives successfully by drilling $6,167 \mathrm{ft}$ total interval of build/lateral section. 
Table 3 Field $Y$ recent wells against DLR wells

\begin{tabular}{lllclll}
\hline Well & $\begin{array}{l}\text { Depth in } \\
(\mathrm{ft})\end{array}$ & $\begin{array}{l}\text { Interval } \\
(\mathrm{ft})\end{array}$ & $\begin{array}{l}\text { ROP } \\
(\mathrm{ft} / \mathrm{h})\end{array}$ & $\begin{array}{l}\text { W\&R } \\
(\mathrm{h})\end{array}$ & BHA & $\begin{array}{l}\text { TQ } \\
(\mathrm{kft} \text { lb) }\end{array}$ \\
\hline $\begin{array}{l}\text { DLR } \\
\text { Well \#2 }\end{array}$ & 4600 & 6318 & 77.1 & 9 & Rotary +2 Stab+DLR@ 550 ft & $3-8$ \\
$\begin{array}{l}\text { DLR } \\
\text { Well \#3 }\end{array}$ & 4590 & 2719 & 140.4 & 17 & Rotary +2 Stab + DLR@ 65 ft & $4-7$ \\
Y1 & 4590 & 6410 & 79.2 & 64 & Rotary +2 stab + Roller Reamer & $5-10$ \\
Y2 & 4594 & 6406 & 81.9 & 28 & Rotary +2 stab +Roller Reamer & $5-8$ \\
\hline
\end{tabular}

- DLR was pulled out in an excellent condition in both runs using Motor \& RSS BHA's.

- No tight spots reported while tripping out the BHAs (Fig. 10).

- The tapered casing production string was run in hole smoothly with no obstructions which confirms the success of the trial test by saving the dedicated stiff reaming run.

- Saved more than $24 \mathrm{~h}$ of rig operations time.

- DLR tool proved its compatibility with motor and RSS system BHA's.

To accurately measure the DLR effect, Caliper log was run in a $61 / 8^{\prime \prime}$ lateral section of one of the wells that deployed this new tool (Fig. 12) compared to its offset well which was drilled recently using the same drilling fluids, BHA type and Drilling unit (Fig. 11). Results proved a slight enlargement (average of 1/16") in the well that deployed the DLR (Fig. 13).

One of the eccentric reamers disadvantages that they may produce a spiral hole in case of high ROP that does not compatible with string RPM that is required to wander the tool off centre to provide non-homogeneous side cutting hole since cutters can be located on only one portion of the circumference of the DLR.

If a wellbore is spiralled (Fig. 14) due to eccentric DLR effect with high ROP and low RPM, the wellbore pass-through area (drift) is calculated according to Lubinski's "crooked hole formula". The pass-through area is a function of the bit diameter and enlarged hole

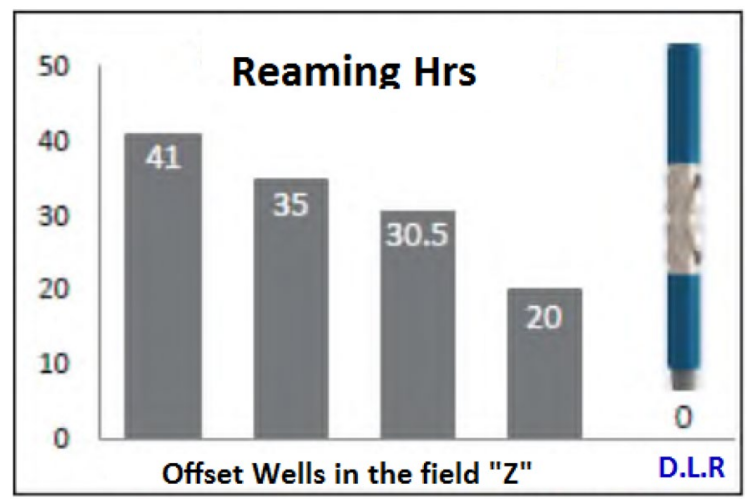

Fig. 10 Reaming Hrs of DLR versus field $Z$ offset wells size by DLR. For example, a 12 1/4" bit and 12 7/16" DLR could produce a pass-through area of $12.343^{\prime \prime}$ (Fig. 15)

Drift $=($ Bit Diameter + DLR $) / 2$

So to avoid well bore spiral problem, we can increase the DLR RPM to catch this high ROP and enlarge the hole with the required degree or increase the depth of cut (DOC) per each rotation or increase the cutters length of DLR as ROP is function of all of them as per the below equation:

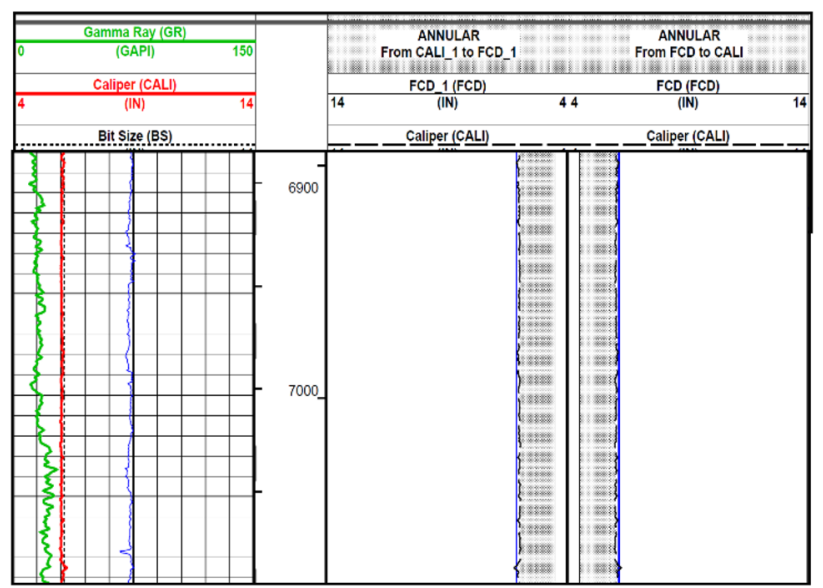

Fig. 11 Offset Well 61/8" lateral section caliper

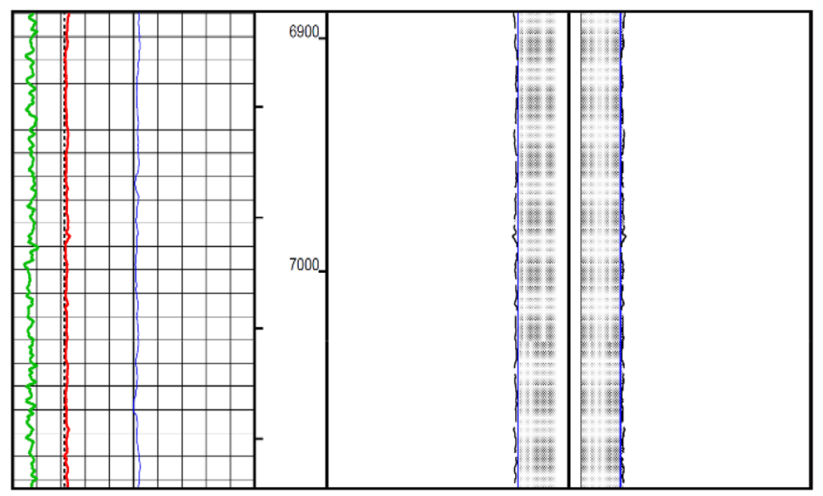

Fig. 12 DLR well $61 / 8^{\prime \prime}$ lateral section caliper 
$\mathrm{ROP}_{(\mathrm{ft} / \mathrm{hr})}=\frac{\text { Cutter Length (in) }}{12} * \mathrm{DLR} \mathrm{RPM} * 60 * \frac{\mathrm{DOC}}{\Delta D}$

Assuming DLR RPM is 60 which may be the maximum surface RPM in case of motor BHA and DOC / hole enlargement $(\Delta D)$ is $1 / 4$ which is matching with this low RPM. (Fig. 16) represents the relation between DLR cutters length increase and ROP. From this graph, we can select DLR with enough cutters length matching with the expected ROP to avoid hole spiral problems.

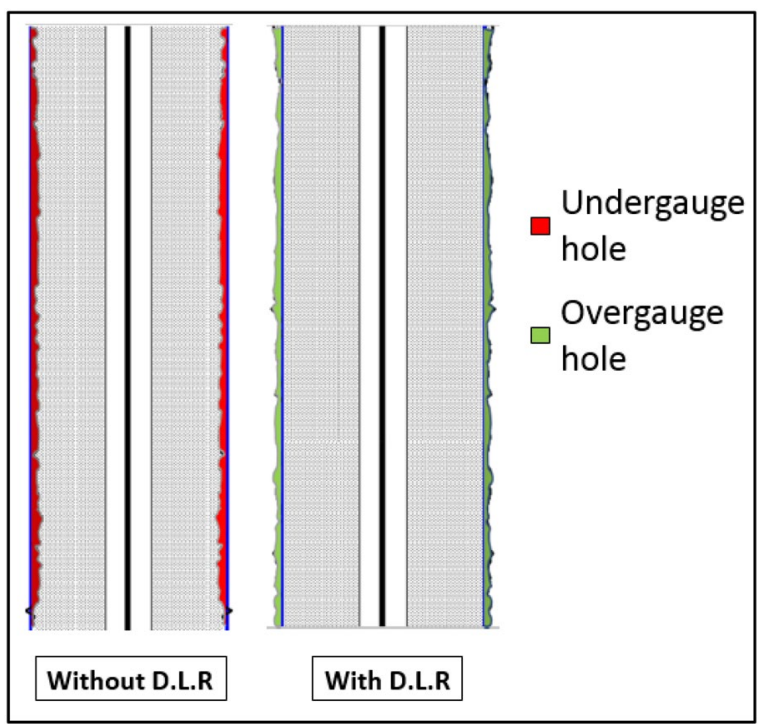

Fig. 13 Slight enlargement after deploying DLR

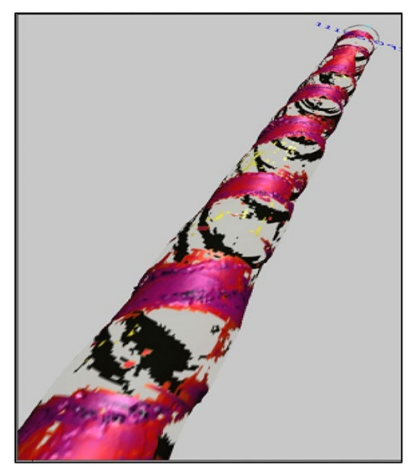

Fig. 14 Spiral borehole as shown by 3D image obtained from a wireline CAST tool in a well in South America

\section{Mathematical model for cutting structure compatibility}

During the design of multiple cutting structures in one drilling system, it is critical to ensure that both cutting structures are compatible and that neither of them is out-drilling the other (Saleh et al. 2014).

One of the success factors during the design of DLR BHA is to study the torque and weight distribution on each cutting structure to emphasize that the cutting element can withstand the applied loads during drilling and tripping phase otherwise accelerated wear will happen and lead to NPT.

Previous work has been done to simulate the load distribution in concentric BHE tools However, limited studies considered to simulate the load distribution between the bit and the eccentric BHE tools. Dykstra et al. in the $90 \mathrm{~s}$ started this work on reamers (Cook et al. 1998; Warren and Dykstra

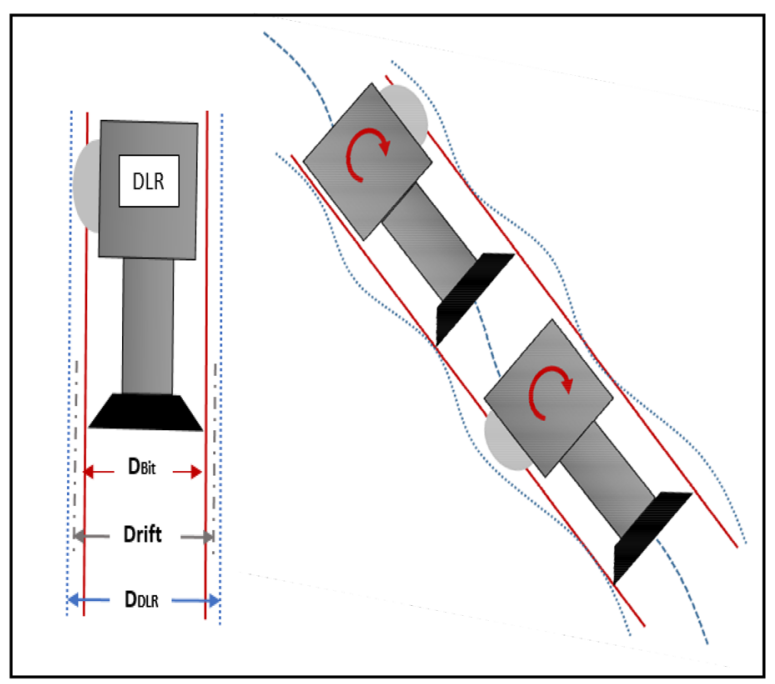

Fig. 15 Relationship between bit diameter and DLR diameter creating a self-limiting system as per Lubinski

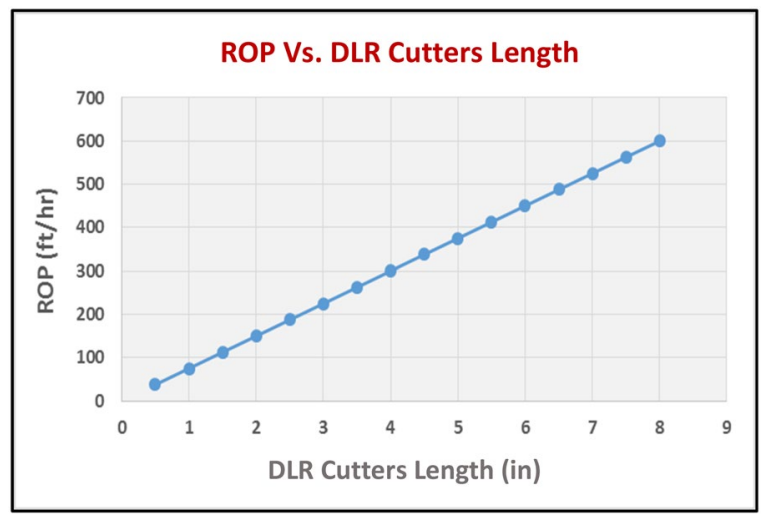

Fig. 16 DLR cutters length versus ROP 
1995). Meyer-Heye studied the stationary and time-dependent load distribution between reamer and bit. His model was based mainly on the mechanical specific energy (MSE) concept (Meyer-Heye et al. 2010) and a complex finite-beam model combined with parameterized force models to simulate dynamic load (Meyer-Heye et al. 2011). The effect of interbedded formations had been discussed by Heisig et al. (2007). Continuous work had been done to improve reamer runs even by deep analysis of case histories or by modelling and simulating their dynamic behaviour (Fahusnaza et al. 2015; Radford et al. 2009; Thomson et al. 2008; Valbuena et al. 2017). Recent paper presented an approach to calculate the drilling energy based on the transient dynamics simulation of the full drilling system. It provides a detailed and holistic view of drilling energy input, propagation and consumption (Chen et al. 2019). A programmatic approach to model ROP for concentric reamers to improve the drilling performance in Gulf of Mexico has been produced by Soares et.al. (2020).

Meyer-Heye et.al model is the base model for MSE as it allows us to study the necessary parameters for optimization algorithms. In this paper, we will depend mainly on its model and slightly modify to match the eccentric motion of DLR.

The stationary model is based on the concept of MSE that relates the energy needed to destroy rock to the destroyed volume of the rock (Teal 1965). The equation to derive the MSE of a bit $\left(\mathrm{MSE}_{\mathrm{B}}\right)$ depends on torque on bit (TOB), weight on bit (WOB), rotation rate (RPM), rate of penetration (ROP) as well as the cross-sectional area drilled by the bit $A_{B}$,

$\mathrm{MSE}_{B}=\frac{\mathrm{WOB}}{A_{B}}+\frac{120 \pi \cdot \mathrm{RPM} \cdot \mathrm{TOB}}{A_{B} \cdot \mathrm{ROP}_{B}}$

Using, $\quad A_{B}=\frac{\pi}{4} \cdot D_{B^{2}}$

Assuming Rotary BHA or RSS BHA while the bit RPM is the same as the string RPM so no need to separate RPMs for bit or DLR. In case of BHA includes downhole motor, drilling bit and DLR would drill at different RPMs, but this case will not investigated in this paper.

Using a DLR does not essentially change the MSE calculation. DLR does not drill the complete hole, but just the difference between main hole and enlarged hole. This changes the cross-sectional area $\left(A_{R}\right)$ and of course weight on reamer (WOR) and torque on reamer (TOR) are used instead of the loads on bit.

As per the tool schematics and depending on the eccentric motion, the cutters are located on only about one fourth of the circumference of the DLR (Fig. 17) so the area drilled per revolution is reduced by this value and due to the lateral

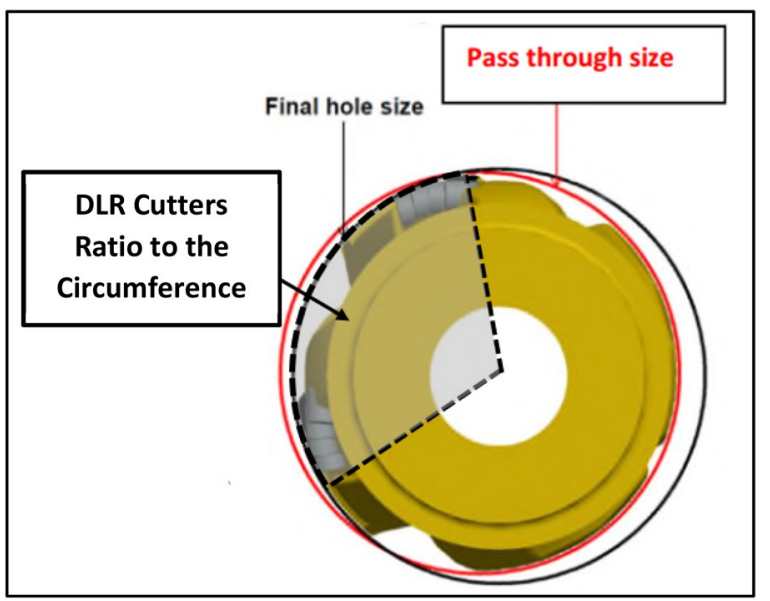

Fig. 17 DLR cutters ration to the circumference

length of cutters that are engaged at the same time to enlarge the hole, the term of ROP of the DLR is different than the bit ROP and dependant on the effective cutters length that are responsible for reaming in the drilling mode so DLR ROP can be expressed by,

$\mathrm{ROP}_{R}=\mathrm{ROP}_{B} * \mathrm{DLR}_{\text {Cutters Length }}$

So the MSE for the DLR can be expressed by:

$\mathrm{MSE}_{R}=\frac{\mathrm{WOR}}{A_{R}}+\frac{120 \pi \cdot \mathrm{RPM} \cdot \mathrm{TOR}}{A_{R} \cdot \mathrm{ROP}_{R}}$

Using, $\quad A_{R}=\frac{\pi}{4} \cdot\left(D_{R}^{2}-D_{B}^{2}\right) \cdot$ Cir. Cutters Ratio

The contribution of the torque on bit (TOB) on the MSE is very high compared to that of the weight on bit (WOB); so we can neglect the contribution of weight factor to be able to cancel the effect of the depth of cut (DOC) on our calculations in the torque and weight ratios and the error produced by this simplifications is not larger than one percent (MeyerHeye et al. 2010). Both MSE equations can be simplified to:

$\mathrm{MSE}_{B}=\frac{120 \pi \cdot \mathrm{RPM} \cdot \mathrm{TOB}}{A_{B} \cdot \mathrm{ROP}_{B}}$

$\mathrm{MSE}_{R}=\frac{120 \pi \cdot \mathrm{RPM} \cdot \mathrm{TOR}}{A_{R} \cdot \mathrm{ROP}_{R}}$

Using,

$\frac{\mathrm{WOB}}{A_{B}} \ll \frac{120 \pi \cdot \mathrm{RPM} \cdot \mathrm{TOB}}{A_{B} \cdot \mathrm{ROP}_{B}}$ 
$\frac{\mathrm{WOR}}{A_{R}} \ll \frac{120 \pi \cdot \mathrm{RPM} \times \mathrm{TOR}}{A_{R} \cdot \mathrm{ROP}_{R}}$

As the drilling efficiency can be derived if we compare the calculated MSE needed to the rock confined compressive strength (CCS) as per Eq. (12):

$=\frac{\mathrm{CCS}}{\mathrm{MSE}}$

CCS is function of the type of rock being drilled and the confining pressure. Combining Eqs. $(8,9,12)$, we can calculate the torque at each tool depending on the drilling efficacy, drilling parameters and rock being drilled.

$\mathrm{TOB}=\frac{A_{B} \cdot \mathrm{ROP}_{B} \cdot \mathrm{CCS}_{B}}{120 \pi \cdot \mathrm{RPM}_{B}}$

$\mathrm{TOR}=\frac{A_{R} \cdot \mathrm{ROP}_{R} \mathrm{CCS}_{R}}{120 \pi \cdot \mathrm{RPM}_{R}}$

The total applied weight by the driller is distributed between the bit and DLR and also the reactive torque produced is the sum of the torque produced at the bit and at DLR. A ratio between the produced torques at DLR to the bit can be obtained by dividing Eq. (14) by Eq. (13).

$\frac{\mathrm{TOR}}{\mathrm{TOB}}=\frac{\mathrm{CCS}_{R}}{\mathrm{CCS}_{B}} \cdot \frac{\eta_{B}}{\eta_{R}} \cdot \frac{A_{R}}{A_{B}} \cdot$ Cutters Length ${ }_{R}$

From Eq. (16), the resulting TOB is dependent on WOB, the bit-specific aggressiveness $\left(\mu_{B}\right)$ and the arm of torque which depends on the drilling Diameter of the bit $D_{B}$ (Meyer-Heye et al. 2010),

TOB $=a_{B} \cdot \mu_{B} \cdot$ WOB

using, $\quad a_{B}=\frac{1}{4} D_{B}$

Depending on the same concept, we can drive the weight and torque on DLR.

$\mathrm{TOR}=a_{R} \cdot \mu_{R} \cdot \mathrm{WOR}$

using, $\quad a_{R}=\frac{1}{4} \frac{D_{R}^{2}-D_{B}^{2}}{D_{R}-D_{B}}$

From Eqs. 16 and 17, a ratio of the weights at DLR to bit can also be calculated as below,

$\frac{\mathrm{WOR}}{\mathrm{WOB}}=\frac{\mathrm{CCS}_{R}}{\mathrm{CCS}_{B}} \cdot \frac{\eta_{B}}{\eta_{R}} \cdot \frac{A_{R}}{A_{B}} \cdot \frac{\mu_{B} \cdot a_{B}}{\mu_{R} \cdot a_{R}} \cdot$ Cuuters Length ${ }_{R}$

When the bit and DLR within the same type of rock, $\mathrm{CCS}_{B}=\mathrm{CCS}_{R}$, at the same efficiency, $\eta_{R}=\eta_{B}$, this would mean both are drilling at the same MSE. In this case, the torque generated at the DLR to the bit is dependent on crosssectional areas drilled by each tool and also on the cutters length of DLR.

$\frac{\mathrm{TOR}}{\mathrm{TOB}}=\frac{A_{R}}{A_{B}} \cdot$ Cuuters Length ${ }_{R}$

$=\frac{\left(D_{R}^{2}-D_{B}^{2}\right) \cdot \text { Cir. Cut Ratio } \cdot \text { Cuuters Length }}{R}$

The weight ratio of the DLR to bit is not only dependent on the drilling diameters but also on the cutting structure represented by the aggressiveness factors $\mu_{R}$ and $\mu_{B}$ so,

$\frac{\mathrm{WOR}}{\mathrm{WOB}}=\frac{A_{R}}{A_{B}} \cdot \frac{\mu_{B} \cdot a_{B}}{\mu_{R} \cdot a_{R}}$.Cuuters Length ${ }_{R}$

$=\frac{\left(D_{R}-D_{B}\right) \cdot \mu_{B} \cdot \text { Cir. Cut Ratio } \cdot \text { Cuuters } L_{R}}{D_{B} \cdot \mu_{R}}$

Figure 18 shows the influence of different formations on the weight and torque ratios of NOV $83 / 8^{\prime \prime} \times 85 / 8^{\prime \prime}$ DLR to $81 \frac{1 / 2}{\prime \prime}$ bit where:

- $\mathrm{D}_{\mathrm{B}}=8.5 \mathrm{in}$.

- $\mathrm{D}_{\mathrm{R}}=8.625$ in.

- Circumference cut ratio $=30 \%$.

- $\mu_{B} / \mu_{R}=1 / 2$.

(Due to the very tapered profile of DLR)

- Total cutters length $=2 * 11=22$ in.

(DLR cutters are double raw).

For the blue line, CCS at the DLR is varied, while the formation at the bit is assumed constant $\mathrm{CCS}_{B}=\mathrm{CCS}_{\text {ref. }}$. For the orange line, the DLR always drills the same formation, $\mathrm{CCS}_{\mathrm{R}}=\mathrm{CCS}_{\text {ref }}$, while CCS at the bit changes.

\section{Results and discussion}

From first implementation all over the world, there are more than twenty successful runs that have been performed to date in hole sizes ranging from $12 \frac{1 / 4^{\prime \prime}}{}$ to $81 / 2^{\prime \prime}$ showing a significant improvement in hole quality which result in cost and time saving.

The weight ratio plot in (Fig. 18) indicates that most of the weight is transferred to the bit unlike DLR due to its higher cross-sectional area compared to the DLR but looking to the torque ratio plot in the same figure, the torque percentage on DLR is significantly higher, in this example up to $40 \%$ in case of $\mathrm{CCS}_{\mathrm{B}} / \mathrm{CCS}_{\mathrm{R}}=1 / 2$. This is the result of 
the greater arm of torque combined with the higher aggressiveness of the DLR.

Field application of DLR BHA showing a significant torque increase (average of 2-3 Klb-ft) compared to the offset wells with the same drilling parameters and this means the average TOR/TOB $=15-30 \%$ which is very close to our calculated model depending on the aggressiveness and confined compressive strength ratios between the bit and DLR.

\section{Conclusions and recommendations}

A DLR tool can be integrated into a rotary and directional motor or RSS BHA. It was designed and optimized based on the eccentric motion concept, and its placement was precisely determined using BHA modelling software to ensure high contact forces to assist in tool engagement with the borehole.

The development of this new tool has added new capability to the industry in the optimization of hole enlargement applications. The benefit is realized in producing a highquality wellbore which have a gauge hole, a smooth wellbore and a wellbore with minimum tortuosity. This high-quality wellbore reduced the number of tight spots and the observed level of drag and overpull showing a significant improvement in the trip out performance in addition to eliminate the need for a dedicated condition run before wire line and casing running increasing the success ratio of wireline logging tools to reach TD and for sure better casing and cement jobs and this sets a more challenging bench marks providing a significant cost saving.

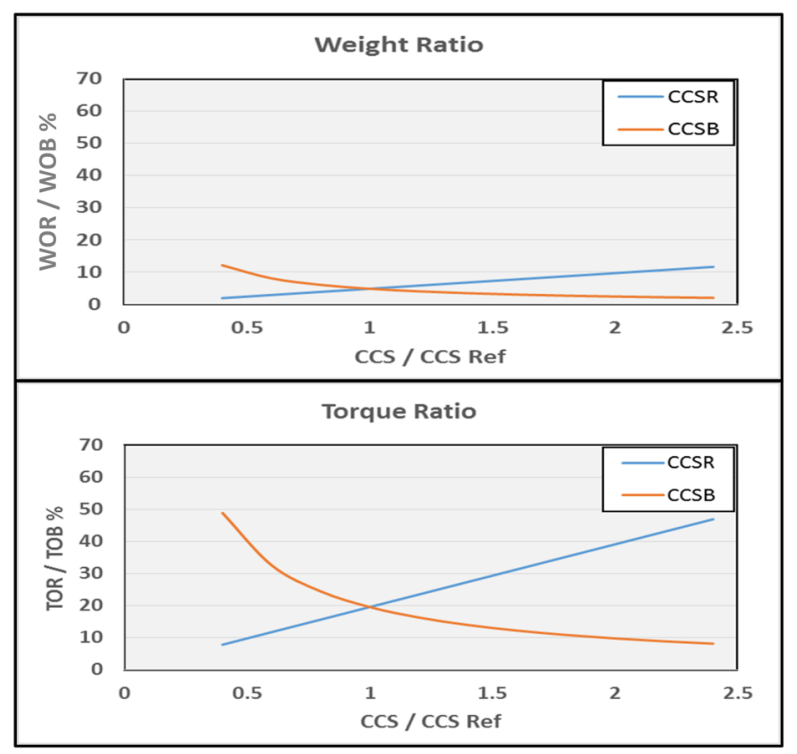

Fig. 18 Torque and weight ratios of DLR to the bit
With the mathematical method derived in this paper, the weight and torque distributions between the DLR and the bit can be calculated. Unlike other types of centric BHE, the weight and torque ratios are significantly affected by circumference cut ratio and active cutters length in addition to the weight distribution in a DLR BHA is dependent on the aggressiveness of the cutting structure at the bit/the DLR and the cross-sectional areas drilled by the DLR and the bit. The torque distribution is dependent on the crosssectional areas.

The mathematical model derived in this paper is based on the assumption of the same RPM at the bit and DLR, so future research could consider the difference in RPM due to down hole motors or any other reasons.

Torque can be used as a real-time indicator for successful tool function; however, it may be not effective in soft or heterogeneous formation.

One of the methods to validate our mathematical model and determine its accuracy level is to conduct an experimental field test-which has not been conducted yet - to get insight to the loads applied at the bit and DLR at the same time. Two identical downhole dynamic measurement tools could be used. The lower measurement tool to be positioned close to the bit and measured the loads and dynamics on the bit. In order to also measure dynamics, weight and torque on the DLR, the second measurement tool was mounted directly above the reamer. In this way, the loads on the DLR could be measured by the difference of the loads at both measurement tools. This system can provide information of tool status, vibration level at the tool, temperature and any other down hole data. All of this information is real-time feedback which will save time and reduce many risks associated due to DLR status while drilling.

Acknowledgements The authors would like to show their appreciation to NOV planning \& operations teams who contributed to safe implementation of DLR BHA.

Funding No funding received for this publication.

Open Access This article is licensed under a Creative Commons Attribution 4.0 International License, which permits use, sharing, adaptation, distribution and reproduction in any medium or format, as long as you give appropriate credit to the original author(s) and the source, provide a link to the Creative Commons licence, and indicate if changes were made. The images or other third party material in this article are included in the article's Creative Commons licence, unless indicated otherwise in a credit line to the material. If material is not included in the article's Creative Commons licence and your intended use is not permitted by statutory regulation or exceeds the permitted use, you will need to obtain permission directly from the copyright holder. To view a copy of this licence, visit http://creativecommons.org/licenses/by/4.0/. 


\section{References}

Barton S, Wedeen R (2010) Unique selection tool enables sientific approach to matched

Chen W, Shen Y, Zahang Z, Bogath C, Harmer R (2019) Understanding drilling system energy beyond MSE. In: SPE annual technical conference, SPE, Canda (196050)

Chowdhury A, Serrano R, Rodrigue W (2019) Pilot bit and reamer matching: real-time downhole data differentiates hybrid drill bit's suitability with concentric reamer in deepwater, Gulf of Mexico application. In: SPE/IADC international drilling conference and exhibition, SPE/IADC, The Netherlands (194060)

Cook J, Doster, M, Dykstra M, McDonald S, Christensen H (1998) Development of steerable ream while drilling technology and its application in high angle wells in GOM. In: IADC/SPE drilling conference, IADC/SPE, Texas (39326)

Fahusnaza N, Soh A, Nair D, Afiq M, James B, Subroto B, Omar T (2015) Advanced FEA modeling delivers solution for designing a dual hole opening BHA for a single directional run. In: SPE/IADC drilling conference and exhibition, SPE/IADC, United Kingdom (173019)

Heisig G, Hood J, Okewunmi S, Robnrett E (2007) Achieving shoe-toshoe drilling performance in hole-opening application with rotarysteerable systems. In: SPE/IADC drilling conference, SPE/IADC, Netherlands (105578)

Huggett T, Hunter G, Alhamad M, Eatough O (2019) Monitoring of uner-reamer function status to confirm in-gauge hole. In: SPE/ IADC drilling international conference and exhibtion, SPE/IADC, Netherlands (194161)

McCarthy J, Rebellon J, Barton S, Rambhai R (2011) Apractical, application-based guide to borehole enlargement tool selection. In: Brasil offshore conference and exhibtion, SPE, Brazil (142431)

Meyer-Heye B, Reckmann H, Ostermeyer G (2010) Weight distribution in reaming while drilling BHAs. In: IADC/SPE drilling conference and exhibition, IADC/SPE, Louisiana (127094)
Meyer-Heye B, Reckmann H, Ostermeyer G (2011) Underreamer dynamics. In: SPE/IADC drilling conference and exhibition. SPE/ IADC, Netherlands (1398983)

Radford S, Li T, Jenkins M (2009) Novel concentric expandable stabilizer results in increased penetration rates and drilling efficiency with reduced vibration. In: SPE/IADC conference and exhibition, SPE/IADC (119534)

Saleh R, Noor GA, Abuahmad Y, Otaibi N, Osman A, Farid M, Ali A, El-Fouly I (2014) Optimizing Saudi aramco reentry drilling operations with the first worldwide combined high dogleg RSS and underreaming drilling system. In: SPE annual technical symposium and exhibitation, SPE, Saudi Arabia (172171)

Soares S, Armenta M, Panchal N (2020) Enhancing reamer drilling performance in deepwater Gulf of Mexico Wells. In: SPE annual conference, SPE, USA (200480)

Teal R (1965) The Concept of Specific Energy in Rock Drilling. Int J Rock Mech Min Sci Geomech 2:57-73

Thomson I, Radford S, Powers J, Shale L, Christensen H, Jenkins M (2008) A systematic approach to a better understanding of the concentric hole-opening process utilizing drilling mechanics and drilling dynamics measurements recorded above and below the reamer. In: IADC/SPE drilling confernce, IADC/SPE, USA (112647)

Valbuena F, Belloso A, Pessier R, Ouestali H (2017) Improving reliability and performance of under-reaming operations. In: SPE/ IADC drilling confernce and exhibition, SPE/IADC, Netherlands (184674)

Warren TM, Dykstra MW (1995) Simultaneous drilling and reaming with fixed blade reamers. In: SPE annual technical conference and exhibition. SPE, USA (30474)

Publisher's Note Springer Nature remains neutral with regard to jurisdictional claims in published maps and institutional affiliations. 